\title{
Propuesta para el desarrollo de la educación superior
}

José Adolfo Araujo-Romagoza

Recibido: 14/03/2014 - Aceptado: 19/04/2014

\section{Resumen}

La Universidad Tecnológica de El Salvador, ante la oportunidad de un nuevo gobierno, presenta la siguiente propuesta para mejorar el subsistema de educación superior. La finalidad de esta propuesta es proporcionarles a las nuevas autoridades una sugerencia de visión y estrategias para el desarrollo de la educación superior, con el propósito de mejorar la relevancia, la calidad y la cobertura. El subsistema de educación superior requiere una actualización y reestructuración para adecuarlo a las necesidades de la educación del nuevo milenio. La propuesta propiamente dicha comprende las características que deben reunir el subsistema de educación superior, la gestión educativa indispensable, la gestión administrativa de soporte y los requisitos para ponerla en marcha.

\section{Palabras clave}

Educación, Educación superior, Currículo-planes de estudio, Planeación educativa, Administración educativa.

\section{Abstract}

Universidad Tecnológica de El Salvador, facing the opportunity of a new government, introduces the following proposal for the improvement of the higher education subsystem in El Salvador. The main goal of this plan is to provide the new governmental authorities with a proposal for a different vision and a new set of strategies for the development of higher education in order to improve its relevance, quality and coverage within the country. The current higher education subsystem calls for an updating and restructuring process that suits the educational needs of the new millennium. The proposal itself comprises the essential features to be complied with by the higher education subsystem, an indispensable educational management, an administrative management support, and all other necessary requirements to implement it accordingly.

\section{Keywords}

Education, Higher Education, Study Plans-Curricula, Planning, Educational Plannning, Educational Administration. 


\section{Propuesta para desarrollar la educación superior}

El subsistema de educación superior puede responder a la demanda de formación de profesionales con un nivel de calificación apropiado, si reúne las siguientes características:

Diversificado. El subsistema de educación superior debe formar profesionales en diferentes áreas, según las necesidades del desarrollo integral, que aproveche todas las potencialidades de producción de bienes y servicios que tiene el país, sin poner en riesgo la vida y que contribuya a disminuir significativamente la pobreza; que forme emprendedores capaces de innovar en su campo profesional y que desarrolle capacidades empresariales para que el graduado pueda crear, promover, asesorar y gestionar empresas. Para ello, es necesario desarrollar programas de orientación educativa y profesional.

Sistémico. El subsistema de educación superior debe tener un enfoque sistémico, que vea la realidad como un todo y no como el montaje de sus partes. Esto implica que el currículo no debe desarrollarse como un conjunto de asignaturas que fragmentan el conocimiento y, por lo tanto, el abordaje de la realidad, pues esto no permite analizar los fenómenos en su contexto y complejidad, en relación con otros fenómenos con los cuales mantiene interacción e interdependencia.

La educación sistémica reconoce que la realidad está organizada en una vasta red de interrelaciones, por lo que el tratamiento que se le debe dar al estudio de los fenómenos debe ser desde diferentes disciplinas. Los estudios por proyectos y por problemas pueden facilitar el desarrollo del currículo.

El cambio consiste en pasar de un modelo mecanicista y reduccionista de la educación a un nuevo modelo centrado en la vida, en la complejidad de la realidad, en la incertidumbre y en el aprendizaje, con un enfoque sistémico.

Relevante. Las carreras profesionales deben responder a las necesidades de la sociedad (nacional e internacional) y de la calidad de vida. La motivación para aprender se logra cuando el estudiante tiene necesidad de adquirir el nuevo aprendizaje, porque le será útil.
El contenido del aprendizaje debe estar relacionado con: las demandas del mercado laboral, el avance de las ciencias y de la tecnología, la recuperación y conservación del medio ambiente, los requerimientos de la globalización $y$, en general, con las demandas de un desarrollo integral, justo y humano.

Competencias generales necesarias. El subsistema de educación superior debe desarrollar competencias generales y de especialidad y un conjunto de valores institucionales y profesionales.

Las competencias generales deben comprender: conocimientos, habilidades y actitudes que le facilitan al graduado un desempeño profesional en diferentes campos.

Se propone el desarrollo de las siguientes competencias generales:

1. Comunicar profesionalmente las ideas en forma oral y escrita.

2. Utilizar adecuadamente las tecnologías de información y comunicación (TIC).

3. Construir un plan de desarrollo personal.

4. Tomar decisiones con fundamentos técnicos.

5. Innovar y ser agente de cambio, promoviendo la cultura y la conservación del medio ambiente.

6. Analizar los procesos globalizadores para enfrentar los cambios.

7. Interpretar textos básicos y comprender vocabulario técnico en el idioma inglés.

8. Practicar una vida digna y respetable en la sociedad a través de la ilustración y la solidaridad.

9. Contribuir a la construcción del conocimiento.

10. Actualizar sus conocimientos en forma permanente.

Algunos valores que las instituciones deben desarrollar en toda su comunidad son los siguientes:

Compromiso, innovación permanente, respeto y pensamiento positivo, liderazgo, solidaridad y trascendencia cultural e integridad.

Ejes transversales de formación. Las instituciones de educación superior deben adoptar ejes transversales del currículo, según su filosofía, carreras e identidad institucional. 
Flexible. El currículo de educación superior no debe ser rígido, porque limita las oportunidades de aprender. La opción es un currículo sin prerrequisitos, con asignaturas optativas, con diversidad de horarios de clases, con múltiples ambientes de aprendizaje, apoyados con una red de aprendizaje y con un soporte administrativo adecuado.

La educación superior debe desarrollarse por proyectos y por problemas reales de la sociedad, de las empresas, del medio ambiente, de la familia, de la persona, tanto en lo individual como social.

De calidad. Las instituciones de educación superior deben formar a sus estudiantes de acuerdo con el perfil profesional que se ha establecido en cada plan de estudios. Para ello, el personal docente, los recursos didácticos y tecnológicos, los ambientes de aprendizaje y el enfoque curricular deben servir para lograr las competencias establecidas en el perfil.

Una estrategia para mejorar la calidad del aprendizaje es que el estudiante adquiera la información en diferentes ambientes, de una diversidad de fuentes, con su propio ritmo y estilo de aprendizaje, en el tiempo que tiene disponible y organizando redes sociales físicas y/o virtuales para construir conocimientos y que asista al aula sólo a desarrollar ejercicios, problemas, casos, proyectos, etc., en los que tenga que aplicar lo aprendido previamente.

La formación por competencias es conveniente, para que estudiantes y docentes no se preocupen solo por el contenido y por las notas, sino por alcanzar el desarrollo de conocimientos, habilidades y actitudes. Los estudiantes no se preocuparán tanto por la nota de aprobación, sino por alcanzar el dominio de las competencias; y el papel de todos los actores del proceso educativo estará orientado hacia ese fin.

Para que los docentes puedan cumplir con sus nuevos papeles, es necesario ejecutar un programa de desarrollo profesional docente no solo para generar metodología pedagógica centrada en el aprendizaje y la evaluación por competencias, sino en la utilización de medios digitales como apoyo didáctico al proceso educativo.

Con cobertura total. Es necesario dar oportunidades de acceso a la educación a los estudiantes que desean continuar sus estudios superiores, adecuando la oferta educativa a las características de la población. Algunos estudiantes pueden dedicarse a tiempo completo al estudio, otros tienen que combinar el estudio con el trabajo, y otros más, que por residir muy lejos de las instituciones o porque su horario de trabajo es incompatible con los horarios de clase que programan las instituciones, no pueden continuar sus estudios; por lo que es necesario desarrollar diversas estrategias de atención, para no dejar fuera del sistema educativo a personas con aspiraciones $y$ deseos de superación.

La flexibilidad del currículo, de los horarios de atención y estrategias de educación a distancia son indispensables, para que cada estudiante haga su propio recorrido por la carrera de estudio en el tiempo que tiene disponible.

Se hace necesaria la utilización de medios digitales para hacer llegar la oferta educativa hasta donde se encuentra el estudiante que no puede asistir regularmente a una institución.

La combinación de modalidades presencial, semipresencial y a distancia es indispensable para facilitar a todos los estudiantes el acceso a la educación superior.

Los resultados de la Prueba de Aprendizajes y Aptitudes para Egresados de Educación Media (Paes) no deben ser determinantes para el ingreso a los estudios de educación superior. Si el sistema ha marginado a muchos estudiantes de una formación básica y media de calidad, por muchas causas, no es justo que las instituciones de educación superior les cierren las puertas. La formación por competencias, una buena motivación para el aprendizaje y un proceso formativo de calidad, que partan del nivel de entrada con el que llegan los estudiantes, orientándolos hacia el dominio de las competencias generales y de especialidad, establecidos en el perfil del profesional que se debe formar, deben ser los compromisos de las instituciones de educación superior.

Integración de la investigación y la proyección social a la docencia. El proceso formativo será enriquecido si la investigación y la proyección social se integran al proceso de aprendizaje de los estudiantes, si se relacionan directamente con el estudio de la carrera; la investigación, para verificar teorías, comprobar hipótesis y para construir el conocimiento; la proyección social, para poner en práctica lo aprendido en favor de grupos sociales socioeconómicamente desfavorecidos. 
Lo anterior no debe limitar la responsabilidad de las instituciones de educación superior, de realizar investigaciones que les permita influir en las políticas de desarrollo del país; en el desarrollo científico y tecnológico; en el mejoramiento de los procesos de producción de bienes y servicios que respeten el medio ambiente y favorezcan la vida.

Investigación para el desarrollo. Para este tipo de investigación es conveniente la búsqueda de financiamiento en las instituciones gubernamentales y demás empresas que sean beneficiadas con los hallazgos de la investigación.

Actividades extracurriculares. El desarrollo de la educación superior debe ser acompañado de un programa de actividades extracurriculares, que contribuya a la formación integral de los estudiantes en áreas como las siguientes: cultural, deportiva, medioambiental, social, económica y aún política.

Es conveniente que las instituciones de educación superior deban tener apertura todo el día y todos los días con programas de extensión y de cultura, para que la población, no solo estudiantil, tenga acceso a actividades educativas y culturales.

\section{Gestión administrativa}

La autonomía, autorregulación y autoorganización de las instituciones de educación superior son necesarias para facilitar la diversificación y flexibilidad de los programas educativos y el desarrollo de estrategias que permitan incrementar la cobertura, la calidad y la relevancia, con formas de entregas presenciales, semipresenciales y a distancia, utilizando las tecnologías de la comunicación e información.

La descentralización de las decisiones institucionales de educación superior es necesaria para atender lo más próximo posible a los estudiantes no solo en trámites administrativos, sino en apoyos académicos que favorezcan el aprendizaje.
Las instituciones de educación superior deben ofrecer condiciones y recursos como satisfactores estudiantiles que procuren el bienestar y las condiciones apropiadas para el desarrollo social e intelectual de los estudiantes.

Para dar acceso a la educación superior, es necesario desarrollar un sistema de crédito educativo, administrado por las instituciones educativas y garantizado por el Gobierno. Sobre este aspecto existe experiencia en El Salvador y en otros países que han dado excelentes resultados.

\section{Requisitos indispensables para ejecutar la propuesta}

Para que la propuesta de desarrollo de la educación superior se haga realidad y llegue a toda la población que demanda educación superior con relevancia, calidad, flexibilidad y centrada en la vida y en el aprendizaje es indispensable que:

1. Se realice un concilio de intereses mínimos entre los principales actores del subsistema de educación superior.

2. Se capacite al personal académico, para que cambie su papel de trasmisor de conocimientos a gestor del aprendizaje.

3. Se capacite al personal administrativo para que simplifique y agilice los procesos educativos.

4. Se evalúe periódicamente y se le dé seguimiento a la ejecución del nuevo modelo de educación superior para garantizar su realización.

\section{Reflexión final}

No ofrecer una educación superior diversificada, sistémica, relevante, de calidad y con una cobertura suficiente es limitar las posibilidades de desarrollo de El Salvador y condenarlo al estancamiento y a la pobreza; es negar el acceso a una mejor calidad de vida. 